\title{
Adaptability and yield potential of new quinoa lines under agro-ecological conditions of Faisalabad-Pakistan
}

\author{
Muhammad Zubair Akram¹,2, Shahzad Maqsood Ahmed Basra', Muhammad Bilal Hafeez ${ }^{1}$, Shahbaz Khan ${ }^{*}$, \\ Samreen Nazeer ${ }^{2}$, Shahid Iqbal ${ }^{3}$, Muhammad Sohail Saddiq ${ }^{4}$, Noreen Zahra ${ }^{5}$ \\ ${ }^{1}$ Department of Agronomy, University of Agriculture, Faisalabad, 38040, Pakistan \\ ${ }^{2}$ Department of Agricultural Genetic Engineering, Faculty of Agricultural Sciences and Technologies, Ömer Halisdemir \\ Üniversitesi, Niğde, 51240, Turkey \\ ${ }^{3}$ Department of Agronomy, Muhammad Nawaz Shareef University of Agriculture (MNSUA), Multan, 60000, Pakistan \\ ${ }^{4}$ Department of Agronomy, Ghazi University, Dera Ghazi Khan, 32200, Pakistan \\ ${ }^{5}$ Department of Botany, University of Agriculture, Faisalabad, 38040, Pakistan
}

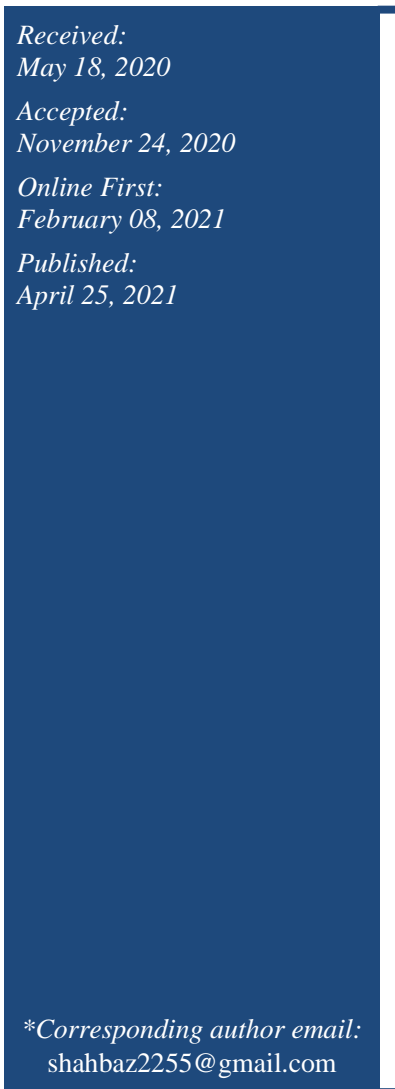

\begin{abstract}
Cultivation of quinoa, a climate-resilient and superfood crop, can be a viable option for food security than conventional food crops. A field experiment was conducted during growing season of 2018-2019 to evaluate 13 elite lines of quinoa. The data regarding stand establishment, phenology of developmental stages, root establishment, leaf biochemical, morphological attributes and seed viability were recorded and analysed. Seed yield was linked with phenological, physiological and morphological traits. Ten lines $(4,6,9,11,15,22,24,30,45$ and 126) seem to be high yielders than check that are originated from New Mexico and Peru. New line Q11 appears to be a high yielder among all the newly introduced lines and standard variety also. Q126 showed the maximum harvesting index. Standard variety UAFQ-7 seems to be higher in root length. Among lines, the duration of crops is also different. Q24 appears to be a short duration crop. Q15 and Q45 appear to be higher in anthocyanin and phenolic contents, respectively. Finding new germplasm having higher yield potential than the check variety will strengthen the popularity of new crops under the agro-climatic conditions of Faisalabad-Pakistan.
\end{abstract}

Keywords: Germplasm, Morphology, Phenology, Quinoa, Root scanner, Seed yield

\section{How to cite this:}

Akram MZ, Basra SMA, Hafeez MB, Khan S, Nazeer S, Iqbal S, Saddiq MS and Zahra N, 2021. Adaptability and yield potential of new quinoa lines under agro-ecological conditions of Faisalabad-Pakistan. Asian J. Agric. Biol. 2021(2):202005301. DOI: https://doi.org/10.35495/ajab.2020.05.301

This is an Open Access article distributed under the terms of the Creative Commons Attribution 3.0 License. (https://creativecommons.org/licenses/by/3.0), which permits unrestricted use, distribution, and reproduction in any medium, provided the original work is properly cited.

\section{Introduction}

Increasing population of the world is demanding an increase in grain production. On the other hand, the world has to shift towards sustainable agriculture in current changing climatic conditions. Crop diversification is not an option but a necessity to safeguard our food supplies, particularly in the context of changing climate. Water and salinity are becoming the most devastating factors that limit agricultural activities due to changing climatic conditions (Radhouane, 2018). Therefore, it is necessary to 
manufacture new plans to cope with these abiotic stress conditions. Growing stress resistance crops can be one of the possible options to meet future food needs (Li et al., 2018). Now we need stress-resistant crops that can survive in such changing climatic conditions without affecting their growth and yield as well. Many halophytic species are present in the Amaranthaceae subfamily. From these halophytes, quinoa (Chenopodium quinoa Willd.) is one of the important crops that is gaining attention nowadays due to its salinity tolerance level, i.e. up to sea level ( $\mathrm{Li}$ et al., 2018; Iqbal et al., 2019). This plant can also cop with water scarcity conditions by reducing its leaves numbers, ultimately reducing leaf surface area and also by the development of a developed rooting system (Fghire et al., 2013). They also develop some special epidermal cell vesicles that act as a water reservoir (Shabala et al., 2012).

Quinoa is an ancient crop that is cultivated in the Andes region for almost 7000 years (Jacobsen et al., 2003; Hellin and Higman, 2005). Recently, quinoa has been widely recommended due to superior nutrition profile outside the Andes. Demand of quinoa is rapidly increasing in high-income countries (Ruiz et al., 2014; Rashid et al., 2018). Peru is the main quinoa producing country in world. Quinoa production is boosted from the last few years over the globe. Many countries are now listed in the quinoa production areas since 2014 but their production is very low as compared to the Andes like United States of America, Europe, Africa and Asia (Zurita-Silva et al., 2014; Bazile et al., 2016).

Quinoa seeds contain 13 to $15 \%$ protein, iron i.e. 81 $\mathrm{mg} / \mathrm{kg}$ and calcium i.e. $874 \mathrm{mg} / \mathrm{kg}$ which are much higher than maize and barley (Vega-Galvez et al., 2010). Repo-Carrasco et al. (2003) stated that quinoa produced in Peru region contained 50\% linoleic acid $(\omega-6)$ and 5\% linolenic acid ( $\omega-3)$. In comparison with protein quality of quinoa with other cereals, quinoa protein contains all essential amino acids while protein quality of wheat and maize is poor although all of these have almost the same protein content (Bertero et al., 2004). Quinoa is easy to digest and is glutenfree, so helpful in the procurement of celiac disease (Bhargava et al., 2006)

Quinoa is now being successfully adapted in Pakistan as it was introduced in 2009. Recently, a variety is also being approved from Federal Seed Certification and Registration Department Islamabad, Pakistan and named as "UAFQ-7". However, being a new crop in the region, more germplasm is required to explore its adaptability and yield potential under the agroclimatic conditions of Faisalabad-Pakistan. Therefore, a field experiment was conducted to evaluate 13 elite lines of quinoa previously screened from USDA germplasm to compare their growth, phenology, root architecture and biochemical attributes at Research Farms Area, University of Agriculture, Faisalabad, Pakistan.

\section{Material and Methods}

During the winter season of 2018-19, a field study was executed to evaluate 13 elite lines of quinoa for growth and productivity at Directorate of Farms, University of Agriculture, Faisalabad-Pakistan (longitude 73.7 E, latitude $31.7 \mathrm{~N}$, and altitude $184 \mathrm{~m}$ ). Seeds of 12 lines of quinoa previously screened from USDA germplasm along with standard check variety UAFQ-7 were collected from Alternate Crops Lab, University of Agriculture, Faisalabad, Pakistan. Details of these genotypes are presented in table 1.

Table-1. Quinoa lines used in the experiment with local code and origin code.

\begin{tabular}{|c|c|c|c|l|}
\hline $\begin{array}{c}\text { Sr. } \\
\#\end{array}$ & $\begin{array}{c}\text { Genotype } \\
\text { local coding }\end{array}$ & Line \# & $\begin{array}{c}\text { Plant } \\
\text { Name }\end{array}$ & \multicolumn{1}{|c|}{ Origin } \\
\hline 1 & Q4 & $\begin{array}{c}\text { Ames } \\
13724\end{array}$ & 18 GR & $\begin{array}{l}\text { United States, } \\
\text { New Mexico }\end{array}$ \\
\hline 2 & Q6 & $\begin{array}{c}\text { Ames } \\
13726\end{array}$ & 49 ALC & $\begin{array}{l}\text { United States, } \\
\text { New Mexico }\end{array}$ \\
\hline 3 & Q9 & $\begin{array}{l}\text { Ames } \\
13729\end{array}$ & $23 P$ & $\begin{array}{l}\text { United States, } \\
\text { New Mexico }\end{array}$ \\
\hline 4 & Q11 & $\begin{array}{c}\text { Ames } \\
13731\end{array}$ & 42 TES & $\begin{array}{l}\text { United States, } \\
\text { New Mexico }\end{array}$ \\
\hline 5 & Q15 & $\begin{array}{c}\text { Ames } \\
13735\end{array}$ & 17 GR & $\begin{array}{l}\text { United States, } \\
\text { New Mexico }\end{array}$ \\
\hline 6 & UAFQ-7 & $\begin{array}{l}\text { Ames } \\
13737\end{array}$ & V7 & $\begin{array}{l}\text { United States, } \\
\text { New Mexico }\end{array}$ \\
\hline 7 & Q22 & $\begin{array}{l}\text { Ames } \\
13742\end{array}$ & 6 P & $\begin{array}{l}\text { United States, } \\
\text { New Mexico }\end{array}$ \\
\hline 8 & Q24 & $\begin{array}{l}\text { Ames } \\
13744\end{array}$ & 409 & $\begin{array}{l}\text { United States, } \\
\text { New Mexico }\end{array}$ \\
\hline 9 & Q30 & $\begin{array}{c}\text { Ames } \\
13750\end{array}$ & A5P & $\begin{array}{l}\text { United States, } \\
\text { New Mexico }\end{array}$ \\
\hline 10 & Q45 & PI 433232 & - & Chile \\
\hline 11 & Q81 & PI 614887 & QQ63 & Chile, Bio-Bio \\
\hline 12 & Q124 & PI 665276 & Line 1376 & Bolivia La Paz \\
\hline 13 & Q126 & PI 665283 & Col.\#6197 & $\begin{array}{l}\text { United States, } \\
\text { Colorado }\end{array}$ \\
\hline
\end{tabular}

The soil of research area was sandy-loam having $\mathrm{pH}$ of 6.8 to 7.3 , electrical conductivity of 3.62 to $3.72 \mathrm{dS}$ $\mathrm{m}^{-1}$, organic matter from 0.88 to $0.91 \%$, total nitrogen contents of 0.07 to $0.08 \%$, exchangeable potassium of 
175 to $177 \mathrm{ppm}$, and available phosphorous of 4.44 to $4.60 \mathrm{ppm}$. The soil of this area is classified as the Lyallpur soil series in Haplic Yermosols in FAO classification. This study was conducted under RCBD design. Two plowing, depth of $12 \mathrm{~cm}$, followed by planking were done for moisture conservation required for emergence. The Chopa method was used for sowing at $5 \mathrm{~kg}$ of seeds per hectare. Seeds were placed at a $15 \mathrm{~cm}$ distance and $75 \mathrm{~cm}$ apart as row spacing. Urea, diammonium phosphate and sulphate of potash were applied as a source of nitrogen, phosphorus and potassium at 75:50:50 kg ha-1 respectively. Half a dose of nitrogen and a full dose of phosphorous and potassium were applied at the time of sowing. Remaining nitrogen was applied with the first irrigation. Insect-pest attack and climatic stress were not observed during the study. Two hoeing were performed manually 45 and 70 days after sowing. The climatic conditions of the study area are semi-arid and sub-tropical. Weather data during the crop duration are shown in table 2.

Table-2. Weather data of growing season of Quinoa crop 2018-19*

\begin{tabular}{|c|c|c|c|c|c|c|c|}
\hline \multirow{2}{*}{ Months } & \multicolumn{3}{|c|}{$\begin{array}{c}\text { Temperature } \\
\left({ }^{\circ} \mathrm{C}\right)\end{array}$} & \multirow{2}{*}{$\begin{array}{c}\text { Relative } \\
\text { Humidity } \\
(\%)\end{array}$} & \multirow{2}{*}{$\begin{array}{c}\text { Total } \\
\text { Rainfall } \\
(\mathrm{mm})\end{array}$} & \multirow{2}{*}{$\begin{array}{c}\text { Sunshine } \\
\text { (hours) }\end{array}$} & \multirow{2}{*}{\begin{tabular}{|c|} 
Wind \\
Speed \\
$(\mathbf{k m}$ \\
$\left.\mathrm{h}^{-1}\right)$ \\
\end{tabular}} \\
\hline & Max & Min & Iean & & & & \\
\hline Jovember & 27.0 & 12.4 & 19.7 & & & & 3.4 \\
\hline Dece & 21.7 & 6. & & & . & 6 & 2.9 \\
\hline January & 19.2 & 7.0 & 13 & & 18.0 & 1.2 & 4.8 \\
\hline February & 20.3 & 9.1 & 14.7 & 79.0 & 64.2 & 6.7 & 4.4 \\
\hline Marcl & 26.0 & 13.8 & 19.9 & & 55.7 & 8.9 & 4.8 \\
\hline April & 33.8 & 19.3 & 26.8 & 42.5 & 31.2 & 9.0 & 5.0 \\
\hline
\end{tabular}

*Source: Agriculture Metrological Cell, Department of Crop Physiology, University of Agriculture, Faisalabad-Pakistan

Data regarding its phenology i.e. emergence, true leaf, four leaves, multiple leaves, bud visible, bus distinct, pyramid, anthesis, milking and total maturity period was obtained by regular visit of the field. Leaf samples were collected at panicle emergence from each plot. These samples were put in the biomedical freezer at a temperature of $-30^{\circ} \mathrm{C}$. Bruinsma's (1963) method was used for the determination of chlorophyll $a, b$ and carotenoids contents of the leaves. The analysis was done on the same day of sampling. Lees and Francis (1971) method was used to obtained anthocyanin contents of the leaves. Anthocyanin from quinoa was observed under UV-V spectrophotometry (Shimadzu UV-1800) at a wavelength of $\lambda=535 \mathrm{~nm}$.
Total anthocyanins (T. A.) $=\frac{A_{535 \mathrm{~nm}} \times \text { dilution factor }}{98.2}$

Anthocyanin contents were expressed in the unit of mg CGE $100 \quad \mathrm{~g}^{-1}$ DW (cyanidin-3-glucoside equivalents per $100 \mathrm{~g}$ dry weight).

For root analysis, sampling was done after 70 days of sowing. Careful sampling was done to avoid the damage and samples were washed with a gentle stream of water. After debris removal, the root samples were scanned and analysed with root scanner by WINRHIZO'S Companion Software for data analysis and visualization for root length, total root surface area, total projected area, volume and average density.

\section{Statistical analysis}

RCBD design was used for the ANOVA. For comparison of individual treatment means Tukey HSD was used at a probability level of 5\% (Steel et al., 1997). Statistix 8.1 was used for the statistical analysis.

\section{Results}

\section{Yield and its attributes}

Significant variations were observed in cultivated lines in comparison with standard variety in plant height, plant biomass, terminal panicle length, terminal panicle, grain yield, thousand-grain weight, grain yield per plant and harvesting index (Table 3 ). Mexican line Q15 attains maximum plant height $(188.87 \mathrm{~cm})$ while Bolivian line Q124 produced the least height of $130.67 \mathrm{~cm}$. Line Q4, Q6, Q9, Q11, Q15, $\mathrm{Q} 45$ and Q81 produced more height as compared to standard variety UAFQ-07 as shown in table 3 . Bolivian line Q124 have more length of terminal panicle $(35.07 \mathrm{~cm})$ that was statistically similar to check variety (UAFQ-07) while maximum terminal panicle grain yield was recorded in Mexican line Q11 (72.20 g) followed by Q4, Q6, Q9 and Q15 to UAFQ07 (Table 3).

Furthermore, line Q4, Q6, Q9, Q11, Q15, Q22 and Q30 produced maximum biological yield in comparison to UAFQ-07 (Table 3). Maximum grain yield was obtained by Mexican line Q11 (80.717 g) while the rest of the lines also produced more grain yield than UAFQ-7 except Q81 and Q124 that produced less yield than UAFQ-07 (Table 3). 
Muhammad Zubair Akram et al.

Table-3. Yield and Yield related traits of 13 quinoa lines

\begin{tabular}{|l|c|c|c|c|c|c|c|}
\hline \multicolumn{1}{|c|}{ Lines } & $\begin{array}{c}\text { Plant } \\
\text { Height } \\
\mathbf{( c m})\end{array}$ & $\begin{array}{c}\text { Plant } \\
\text { Biomass } \\
\mathbf{( g )}\end{array}$ & $\begin{array}{c}\text { Terminal } \\
\text { Panicle } \\
\text { Length }(\mathbf{c m})\end{array}$ & $\begin{array}{c}\text { Terminal } \\
\text { Panicle Grain } \\
\text { Yield }(\mathbf{g})\end{array}$ & $\begin{array}{c}\text { Grain } \\
\text { Yield (g) }\end{array}$ & $\begin{array}{c}\text { 1000 } \\
\text { Grain } \\
\text { Weight }(\mathbf{g})\end{array}$ & $\begin{array}{c}\text { Harvestin } \\
\text { g Index } \\
(\%)\end{array}$ \\
\hline Q4 & $157.60 \mathrm{~d}-\mathrm{f}$ & $173.03 \mathrm{c}$ & $27.77 \mathrm{ab}$ & $69.13 \mathrm{ab}$ & $73.83 \mathrm{ab}$ & $3.287 \mathrm{a}-\mathrm{c}$ & $42.7 \mathrm{c}$ \\
\hline Q6 & $165.63 \mathrm{~cd}$ & $169.43 \mathrm{c}$ & $30.03 \mathrm{ab}$ & $61.53 \mathrm{~b}$ & $69.23 \mathrm{bc}$ & $3.337 \mathrm{a}-\mathrm{c}$ & $40.9 \mathrm{~cd}$ \\
\hline Q9 & $172.60 \mathrm{bc}$ & $193.33 \mathrm{~b}$ & $24.17 \mathrm{~b}$ & $48.67 \mathrm{c}$ & $65.23 \mathrm{bc}$ & $3.143 \mathrm{c}$ & $33.9 \mathrm{ef}$ \\
\hline Q11 & $175.67 \mathrm{~b}$ & $211.67 \mathrm{a}$ & $31.50 \mathrm{ab}$ & $75.20 \mathrm{a}$ & $80.717 \mathrm{a}$ & $3.163 \mathrm{c}$ & $38.1 \mathrm{c}-\mathrm{e}$ \\
\hline Q15 & $188.87 \mathrm{a}$ & $191.03 \mathrm{~b}$ & $24.90 \mathrm{~b}$ & $48 \mathrm{c}$ & $64.52 \mathrm{~cd}$ & $3.273 \mathrm{a}-\mathrm{c}$ & $33.8 \mathrm{ef}$ \\
\hline *UAFQ-07 & $152.73 \mathrm{f}$ & $156.27 \mathrm{de}$ & $33.13 \mathrm{a}$ & $45.07 \mathrm{~cd}$ & $55.83 \mathrm{e}$ & $3.233 \mathrm{a}-\mathrm{c}$ & $35.8 \mathrm{de}$ \\
\hline Q22 & $149.80 \mathrm{f}$ & $173.53 \mathrm{c}$ & $28.27 \mathrm{ab}$ & $40.70 \mathrm{c}-\mathrm{e}$ & $59.62 \mathrm{de}$ & $3.327 \mathrm{a}-\mathrm{c}$ & $34.4 \mathrm{e}$ \\
\hline Q24 & $140.43 \mathrm{~g}$ & $153.5 \mathrm{e}$ & $25.13 \mathrm{~b}$ & $36.93 \mathrm{~d}-\mathrm{f}$ & $75.33 \mathrm{ab}$ & $3.170 \mathrm{bc}$ & $49.1 \mathrm{~b}$ \\
\hline Q30 & $163.40 \mathrm{de}$ & $165.17 \mathrm{~cd}$ & $31.03 \mathrm{ab}$ & $36.23 \mathrm{ef}$ & $60.67 \mathrm{de}$ & $3.230 \mathrm{a}-\mathrm{c}$ & $36.8 \mathrm{de}$ \\
\hline Q45 & $155.87 \mathrm{ef}$ & $150.2 \mathrm{e}$ & $31.20 \mathrm{ab}$ & $28.67 \mathrm{fg}$ & $60.67 \mathrm{de}$ & $3.357 \mathrm{a}-\mathrm{c}$ & $40.4 \mathrm{~cd}$ \\
\hline Q81 & $157.40 \mathrm{~d}-\mathrm{f}$ & $124.73 \mathrm{f}$ & $24.20 \mathrm{~b}$ & $29.83 \mathrm{f}$ & $40.93 \mathrm{f}$ & $3.403 \mathrm{a}$ & $32.9 \mathrm{ef}$ \\
\hline Q124 & $130.67 \mathrm{~h}$ & $109.07 \mathrm{~g}$ & $35.07 \mathrm{a}$ & $21.17 \mathrm{~g}$ & $30.70 \mathrm{~g}$ & $3.383 \mathrm{ab}$ & $28.1 \mathrm{f}$ \\
\hline Q126 & $140.6 \mathrm{~g}$ & $105 \mathrm{~g}$ & $24.90 \mathrm{~b}$ & $44.17 \mathrm{c}-\mathrm{e}$ & $73.13 \mathrm{~cd}$ & $3.447 \mathrm{a}$ & $62.6 \mathrm{a}$ \\
\hline HSD & 8.6571 & 9.8253 & 7.9340 & 8.5447 & 7.7291 & 0.2175 & 0.584 \\
\hline
\end{tabular}

*Standard check variety $=$ UAFQ-07

Colorado line Q126 showed the highest 1000-grain weight (3.447 g) and Q4, Q6, Q15, Q22, Q45, Q81, Q124 and Q126 are leading lines than UAFQ-07 (Table 3). In the case of harvesting index, Colorado line Q126 has maximum harvesting index and the rest of the others Q4, Q6, Q11, Q24, Q30 and Q45 have higher harvesting index than UAFQ-07 (Table 3).

\section{Physiological traits}

Maximum chlorophyll $a$ contents were observed in Mexican line Q30 (Table 4). Line Q4, Q15 and Q30 observed to be higher in chlorophyll $a$ contents than UAFQ-07 (Table 4). Chlorophyll $b$ contents were found maximum in UAFQ-07 (Table 4). Total chlorophyll contents were also recorded maximum in UAFQ-07 (Table 4). Carotenoids were present in maximum amounts in Bolivian line Q124. Line Q24 and Q126 have higher carotenoids contents than UAFQ-07 (Table 4). Phenolic contents were maximum in the Chilean line Q45. Anthocyanin contents were also observed in leaves and results showed that Mexican line Q15 produced maximum anthocyanin contents while Q6, Q9, Q11, Q22, and Q24 also showed more anthocyanin contents as compared to UAFQ-7 (Table 4).

\section{Phonological attributes}

Quinoa lines showed significantly varied maturity period ranges from 128 to 147 days. Minimum crop duration was observed in Mexican line Q24 (128 days) and maximum crop duration was observed in Mexican line Q11 (Table 5). The rest of the lines has almost the same crop duration as the standard variety UAFQ-07. In the quinoa field, 10 defined stages have been observed. Days to emergence, true leave, four leaves, multiple leaves, bud visible, bud distinct, pyramid, flowering, milking and seed setting (Table 5).

\section{Root Scanning}

Roots were sampled at the anthesis stage. The maximum root length was found in UAFQ-07 (Table 6). The maximum surface area was seen in the Mexican line Q15 (Table 6). Four lines Q4, Q15, Q24 and Q124 have high surface area than UAFQ-07 (Table 6). The projected area was observed maximum in Mexican line Q15 followed by standard UAFQ-07 (Table 6). Root volume was highest in UAFQ-07 while minimum in Mexican line Q6 (Table 6). Chilean line Q81 showed more average density than UAFQ-07 (Table 6). Lines Q6, Q9, Q11, Q22, Q30, Q45, Q81, Q124 and Q126 produced high average density than our UAFQ-07 (Table 6). 
Table-4. Physiological data of 13 quinoa lines

\begin{tabular}{|c|c|c|c|c|c|c|}
\hline Lines & $\begin{array}{c}\text { Chlo a } \\
\text { (mg/g Fwt) }\end{array}$ & $\begin{array}{c}\text { Chlo b } \\
\text { (mg/g Fwt) }\end{array}$ & $\begin{array}{c}\text { Total chlo } \\
\text { (mg/g Fwt) }\end{array}$ & $\begin{array}{c}\text { Carotenoids } \\
\text { (mg/g Fwt) }\end{array}$ & $\begin{array}{l}\text { Phenolic } \\
(\mu \mathrm{g} / \mathrm{g} \text { Fwt })\end{array}$ & Anthocyanin \\
\hline Q4 & $0.0353 \mathrm{ab}$ & $0.0973 \mathrm{ab}$ & $0.1330 \mathrm{ab}$ & $1.8420 \mathrm{a}$ & $0.5580 \mathrm{a}-\mathrm{c}$ & $0.9063 \mathrm{a}-\mathrm{c}$ \\
\hline Q6 & $0.0273 \mathrm{ab}$ & $0.0850 \mathrm{ab}$ & $0.1117 \mathrm{ab}$ & $1.8800 \mathrm{a}$ & $0.4763 \mathrm{bc}$ & $1.2160 \mathrm{ab}$ \\
\hline Q9 & $0.0180 \mathrm{~b}$ & $0.0760 \mathrm{~b}$ & $0.0940 \mathrm{~b}$ & $1.6623 \mathrm{a}$ & $0.6363 \mathrm{ab}$ & $1.2307 \mathrm{ab}$ \\
\hline Q11 & $0.0217 \mathrm{ab}$ & $0.0773 \mathrm{~b}$ & $0.0990 \mathrm{ab}$ & $1.7547 \mathrm{a}$ & $0.5753 \mathrm{a}-\mathrm{c}$ & $1.0640 \mathrm{a}-\mathrm{c}$ \\
\hline Q15 & $0.0367 \mathrm{ab}$ & $0.1083 \mathrm{ab}$ & $0.1450 \mathrm{ab}$ & $2.7117 \mathrm{a}$ & $0.5693 \mathrm{a}-\mathrm{c}$ & $1.4460 \mathrm{a}$ \\
\hline *UAFQ-07 & $0.0353 \mathrm{ab}$ & $0.1197 \mathrm{a}$ & $0.1547 \mathrm{a}$ & $2.7130 \mathrm{a}$ & $0.5843 \mathrm{a}-\mathrm{c}$ & $1.0350 \mathrm{a}-\mathrm{c}$ \\
\hline Q22 & $0.0287 \mathrm{ab}$ & $0.0860 \mathrm{ab}$ & $0.1147 \mathrm{ab}$ & $2.3480 \mathrm{a}$ & $0.5310 \mathrm{a}-\mathrm{c}$ & $1.1600 \mathrm{ab}$ \\
\hline Q24 & $0.0220 \mathrm{ab}$ & $0.0950 \mathrm{ab}$ & $0.1170 \mathrm{ab}$ & $2.9610 \mathrm{a}$ & $0.5400 \mathrm{a}-\mathrm{c}$ & $1.1563 \mathrm{ab}$ \\
\hline Q30 & $0.0400 \mathrm{a}$ & $0.1100 \mathrm{ab}$ & $0.1500 \mathrm{ab}$ & $2.2350 \mathrm{a}$ & $0.5357 \mathrm{a}-\mathrm{c}$ & $0.8403 \mathrm{bc}$ \\
\hline Q45 & $0.0293 \mathrm{ab}$ & $0.0910 \mathrm{ab}$ & $0.1207 \mathrm{ab}$ & $1.7547 \mathrm{a}$ & $0.6993 \mathrm{a}$ & $0.8917 \mathrm{a}-\mathrm{c}$ \\
\hline Q81 & $0.0283 \mathrm{ab}$ & $0.0880 \mathrm{ab}$ & $0.1160 \mathrm{ab}$ & $1.8677 \mathrm{a}$ & $0.5643 \mathrm{a}-\mathrm{c}$ & $0.5217 \mathrm{c}$ \\
\hline Q124 & $0.0297 \mathrm{ab}$ & $0.1013 \mathrm{ab}$ & $0.1310 \mathrm{ab}$ & $3.0870 \mathrm{a}$ & $0.4347 \mathrm{c}$ & $0.7333 \mathrm{bc}$ \\
\hline Q126 & $0.0233 \mathrm{ab}$ & $0.1113 \mathrm{ab}$ & $0.1340 \mathrm{ab}$ & $3.0330 \mathrm{a}$ & $0.5927 \mathrm{a}-\mathrm{c}$ & $0.6850 \mathrm{bc}$ \\
\hline HSD & 0.0216 & 0.0417 & 0.0570 & 1.6585 & 0.1756 & 0.5892 \\
\hline
\end{tabular}

$*$ Standard check variety $=$ UAFQ-07

Table-5. Phonological data regarding 13 quinoa lines

\begin{tabular}{|c|c|c|c|c|c|c|c|c|c|c|c|}
\hline Lines & $\begin{array}{c}\text { Days to } \\
\text { emergence }\end{array}$ & \begin{tabular}{|c|}
$\begin{array}{c}\text { Days to } \\
\text { true } \\
\text { leaf }\end{array}$ \\
\end{tabular} & \begin{tabular}{|c|}
$\begin{array}{c}\text { Days to } \\
\text { four } \\
\text { leaf }\end{array}$ \\
\end{tabular} & $\begin{array}{c}\text { Days to } \\
\text { multiple } \\
\text { leaf }\end{array}$ & $\begin{array}{c}\text { Days to } \\
\text { bud } \\
\text { initiation }\end{array}$ & $\begin{array}{c}\text { Days to } \\
\text { bud } \\
\text { distinct }\end{array}$ & $\begin{array}{c}\text { Days to } \\
\text { pyramid }\end{array}$ & $\begin{array}{l}\text { Days to } \\
\text { flowering }\end{array}$ & $\begin{array}{l}\text { Days to } \\
\text { milking }\end{array}$ & $\begin{array}{l}\text { Days to } \\
\text { seed set }\end{array}$ & $\begin{array}{c}\text { Days to } \\
\text { maturity }\end{array}$ \\
\hline Q4 & $5 a$ & $6 a$ & $14 \mathrm{ab}$ & $24 \mathrm{ab}$ & $44 \mathrm{f}$ & $57 \mathrm{c}$ & $70 \mathrm{~b}$ & $80 \mathrm{de}$ & $109 \mathrm{de}$ & $125 \mathrm{~g}$ & $137 \mathrm{e}$ \\
\hline Q6 & $6 \mathrm{a}$ & $8 \mathrm{a}$ & $13 \mathrm{~b}$ & $21 \mathrm{c}$ & $46 \mathrm{e}$ & $53 \mathrm{e}$ & $64 \mathrm{~d}$ & $77 \mathrm{~g}$ & $106 \mathrm{f}$ & $124 \mathrm{~h}$ & $146 \mathrm{ab}$ \\
\hline Q9 & $6 a$ & $7 a$ & $14 \mathrm{ab}$ & $24 \mathrm{ab}$ & $46 \mathrm{e}$ & $55 \mathrm{~d}$ & $66 \mathrm{~cd}$ & $81 \mathrm{~cd}$ & $115 \mathrm{~b}$ & $132 \mathrm{~b}$ & $143 \mathrm{~d}$ \\
\hline Q11 & $7 a$ & $6 a$ & $13 \mathrm{~b}$ & $24 a b$ & $47 \mathrm{de}$ & $55 \mathrm{~d}$ & $68 \mathrm{bc}$ & $80 \mathrm{de}$ & $105 \mathrm{f}$ & $124 \mathrm{~h}$ & $147 \mathrm{a}$ \\
\hline Q15 & $5 a$ & $6 a$ & $14 \mathrm{ab}$ & $23 \mathrm{a}-\mathrm{c}$ & $44 \mathrm{f}$ & $53 \mathrm{e}$ & $65 \mathrm{~d}$ & $78 \mathrm{fg}$ & $113 \mathrm{c}$ & $128 \mathrm{~d}$ & $145 \mathrm{bc}$ \\
\hline *UAFQ-07 & $5 a$ & $6 \mathrm{a}$ & $14 \mathrm{ab}$ & $23 a-c$ & $51 \mathrm{c}$ & $58 \mathrm{bc}$ & $68 \mathrm{bc}$ & 79 ef & $110 \mathrm{~d}$ & $126 \mathrm{f}$ & $145 \mathrm{bc}$ \\
\hline Q22 & $5 a$ & $7 \mathrm{a}$ & $15 \mathrm{a}$ & $23 \mathrm{a}-\mathrm{c}$ & $48 \mathrm{~d}$ & $59 \mathrm{~b}$ & $70 \mathrm{~b}$ & $82 \mathrm{c}$ & $108 \mathrm{e}$ & $122 \mathrm{i}$ & $145 \mathrm{bc}$ \\
\hline Q24 & $5 a$ & $6 \mathrm{a}$ & $14 \mathrm{ab}$ & $24 \mathrm{ab}$ & $48 \mathrm{~d}$ & $58 \mathrm{bc}$ & $70 \mathrm{~b}$ & $82 \mathrm{c}$ & $102 \mathrm{~g}$ & $115 \mathrm{j}$ & $128 \mathrm{f}$ \\
\hline Q30 & $6 \mathrm{a}$ & $7 \mathrm{a}$ & $15 \mathrm{a}$ & $25 \mathrm{a}$ & $46 \mathrm{e}$ & $59 \mathrm{~b}$ & $68 \mathrm{bc}$ & $78 \mathrm{fg}$ & $115 \mathrm{~b}$ & $129 \mathrm{c}$ & $144 \mathrm{~cd}$ \\
\hline Q45 & $5 \mathrm{a}$ & $6 a$ & $14 \mathrm{ab}$ & $25 \mathrm{ab}$ & $57 \mathrm{a}$ & $65 \mathrm{a}$ & $76 a$ & $87 \mathrm{a}$ & $110 \mathrm{~d}$ & $125 \mathrm{~g}$ & $145 \mathrm{bc}$ \\
\hline Q81 & $6 a$ & $6 a$ & $14 \mathrm{ab}$ & $25 \mathrm{a}$ & $57 \mathrm{a}$ & $66 \mathrm{a}$ & $77 \mathrm{a}$ & $87 \mathrm{a}$ & $105 \mathrm{f}$ & $127 \mathrm{e}$ & $144 \mathrm{~cd}$ \\
\hline Q124 & $6 \mathrm{a}$ & $7 \mathrm{a}$ & $15 \mathrm{a}$ & 22 bc & $54 \mathrm{~b}$ & $57 \mathrm{c}$ & $69 \mathrm{~b}$ & $81 \mathrm{~cd}$ & $117 \mathrm{a}$ & $132 \mathrm{~b}$ & $145 \mathrm{bc}$ \\
\hline Q126 & $6 a$ & $7 \mathrm{a}$ & $15 \mathrm{a}$ & $22 b c$ & $55 \mathrm{~b}$ & $65 \mathrm{a}$ & $77 \mathrm{a}$ & $84 \mathrm{~b}$ & $115 \mathrm{~b}$ & $135 \mathrm{a}$ & $146 \mathrm{ab}$ \\
\hline HSD & 2.9886 & 2.9110 & 1.8066 & 2.8714 & 1.7255 & 1.8223 & 2.4636 & 1.4357 & 1.4357 & 0.8289 & 1.4357 \\
\hline
\end{tabular}

*DAS: Days after sowing; *DAE: Days after emergence; *Standard check variety= UAFQ-07

Table-6. Root scanning of 13 quinoa lines in root scanner by using WinRHIZO software

\begin{tabular}{|l|c|c|c|c|c|}
\hline \multicolumn{1}{|c|}{ Lines } & $\begin{array}{c}\text { Root Length } \\
(\mathbf{c m})\end{array}$ & $\begin{array}{c}\text { Projected area } \\
\left(\mathbf{c m}^{\mathbf{2}}\right)\end{array}$ & $\begin{array}{c}\text { Surface Area } \\
\left(\mathbf{c m}^{\mathbf{2}}\right)\end{array}$ & $\begin{array}{c}\text { Volume } \\
\left(\mathbf{c m}^{\mathbf{3}}\right)\end{array}$ & $\begin{array}{c}\text { Average Density } \\
(\mathbf{m m})\end{array}$ \\
\hline Q4 & $479.83 \mathrm{ab}$ & $27.93 \mathrm{a}-\mathrm{c}$ & $90.89 \mathrm{a}$ & $1.1107 \mathrm{a}$ & $0.5173 \mathrm{c}$ \\
\hline Q6 & $124.61 \mathrm{ef}$ & $20.07 \mathrm{a}-\mathrm{e}$ & $32.80 \mathrm{de}$ & $0.7353 \mathrm{a}$ & $0.7260 \mathrm{a}-\mathrm{c}$ \\
\hline Q9 & $83.70 \mathrm{f}$ & $11.02 \mathrm{e}$ & $50.49 \mathrm{bc}$ & $0.9607 \mathrm{a}$ & $1.1123 \mathrm{a}-\mathrm{c}$ \\
\hline Q11 & $78.11 \mathrm{f}$ & $14.71 \mathrm{de}$ & $30.45 \mathrm{de}$ & $0.8590 \mathrm{a}$ & $1.1863 \mathrm{ab}$ \\
\hline Q15 & $513.56 \mathrm{ab}$ & $30.87 \mathrm{a}$ & $92.13 \mathrm{a}$ & $1.0343 \mathrm{a}$ & $0.5150 \mathrm{c}$ \\
\hline *UAFQ-07 & $578.30 \mathrm{a}$ & $30.27 \mathrm{ab}$ & $80.49 \mathrm{a}$ & $1.1920 \mathrm{a}$ & $0.5567 \mathrm{bc}$ \\
\hline Q22 & $273.74 \mathrm{~d}$ & $18.25 \mathrm{~b}-\mathrm{e}$ & $55.93 \mathrm{~b}$ & $0.8250 \mathrm{a}$ & $1.2360 \mathrm{a}$ \\
\hline Q24 & $508.36 \mathrm{ab}$ & $28.58 \mathrm{a}-\mathrm{c}$ & $90.12 \mathrm{a}$ & $1.0870 \mathrm{a}$ & $0.5090 \mathrm{c}$ \\
\hline Q30 & $124.89 \mathrm{ef}$ & $13.97 \mathrm{de}$ & $40.60 \mathrm{~cd}$ & $0.9187 \mathrm{a}$ & $0.8753 \mathrm{a}-\mathrm{c}$ \\
\hline Q45 & $327.19 \mathrm{~cd}$ & $22.30 \mathrm{a}-\mathrm{e}$ & $22.26 \mathrm{e}$ & $1.0843 \mathrm{a}$ & $0.8140 \mathrm{a}-\mathrm{c}$ \\
\hline Q81 & $232.60 \mathrm{de}$ & $22.48 \mathrm{a}-\mathrm{e}$ & $40.71 \mathrm{~cd}$ & $1.0797 \mathrm{a}$ & $1.2370 \mathrm{a}$ \\
\hline Q124 & $416.59 \mathrm{bc}$ & $24.77 \mathrm{a}-\mathrm{d}$ & $81.56 \mathrm{a}$ & $0.9710 \mathrm{a}$ & $1.1300 \mathrm{a}-\mathrm{c}$ \\
\hline Q126 & $305.26 \mathrm{~cd}$ & $17.68 \mathrm{c}-\mathrm{e}$ & $24.33 \mathrm{e}$ & $0.8667 \mathrm{a}$ & $0.6703 \mathrm{a}-\mathrm{c}$ \\
\hline HSD & 133.46 & 12.299 & 13.909 & 0.6564 & 0.6315 \\
\hline
\end{tabular}




\section{Discussion}

A field experiment was conducted to evaluate 13 elite lines of quinoa regarding stand establishment, phenology of developmental stages, root establishment, leaf biochemical, morphological attributes and seed viability. Significant variations were observed in cultivated lines in comparison with standard variety in plant height, plant biomass, terminal panicle length, terminal panicle, grain yield, thousand-grain weight, grain yield per plant and harvesting index. The genotype and location of the plant directly affect the growth behaviour of a crop plant. The plant height of quinoa varied significantly in the present study. Pulvento et al. (2010) also confirmed that under different geographical regions the quinoa plant gains different height. Quinoa completes its life cycle almost in six months, which may be different in different regions (Sajjad et al., 2014). The present study shows that the Mexican line gained more height (Table 3). Our findings are also in line Iqbal et al. (2017) findings who reported that plant of quinoa can grow 60 to $120 \mathrm{~cm}$ which depends upon sowing density as well as on inherent genetics (Iqbal et al., 2017).

From a botanical view, quinoa is considered as a dicotyledonous plant ranges in height $(0.3-3 \mathrm{~m})$. The rate of production of lateral branches directly interlinked with genotype and environmental conditions. Chlorophyll contents are directly linked with the photosynthetic system of plants and it gave the most reliable information about the physiological status of plants (Khosravinejad et al., 2008; Meloni et al., 2008). According to Parida and Das (2005) study, total chlorophyll contents were increased during its vegetative phase and pronounced decrease in the chlorophyll content as the crop reaches its maturity. Similarly, in current work 12 different lines of quinoa were grown to evaluate photosynthetic pigments of quinoa plants. Among all cultivars three lines showed better performance in chlorophyll in comparison with standard variety UAFQ-7. While Bolivian line Q124 produces a high concentration of carotenoids. Phenolics are considered as secondary metabolites in plants (Brend et al., 2012). In current work, it was observed that total phenolic contents were high in the Chilean line Q45 to UAFQ-7. Twelve lines were grown along with one standard variety UAFQ-7 and resulted that different lines have different crop duration (Table 4).
Finding of the current experimentation (Table 5) are also supported by Basra et al. (2014) who stated that bud appears after 30 to 35 days of emergence in quinoa. Anthesis stage was depends upon the ecotype. Anthesis stage was completed as extrusion of anthers completes (Sosa-Zuniga et al., 2017). It matures in 131-140 days after sowing (Basra et al., 2014). All lines attained multiple leaf stage within 21 to 25 days and in most of line pyramid buds appeared 64 to 79 days after emergence. These variations could be linked with different origins of quinoa lines (Curti et al., 2016).

From the last few centuries, studies on root growth have been increased rapidly and significant progress has been observed in evaluating root morphology (Razaq et al., 2017). WinRHIZO software is recommended to explained study of root morphology and architecture. The competitive ability of a plant can be determined through an efficient attainment system of under-ground resources of water. In current research maximum root length was observed in our standard variety UAFQ-7 which confirmed the potential adaptability of this line. Roots are personified as nonvisible parts of plants in biology (Waisel et al., 2002) which are represented in the activity of microorganisms (Gregory, 2006). It was observed from current work that four lines $(\mathrm{Q} 4, \mathrm{Q} 15$, Q24 and Q124) have elevated surface area than standard UAFQ-7 (Table 6).

Grain yield showed a positive correlation and highly significant with the harvest index. So, it exposed that selection on the bases of harvest index for the improvement of yield are useful. Plant height showed a positive and highly significant correlation with grain yield. According to the positive correlation, selection can be made on the bases of plant height for the betterment of grain yield of understudied lines (Table 7). A positive and highly significant correlation is observed between the plant biomass and grain yield. It showed that selection on the bases of plant height for the improvement of grain yield for the understudied line will be beneficiary (Table 7). These results are as similar as reported by Bhargava et al. (2008). Other traits such as carotenoids contents of leaves, root length, days to flowering, days to maturity, total chlorophyll contents, terminal panicle length and thousand-grain weight had shown negative but nonsignificant correlation with grain yield of understudied lines (Table 7). 
Muhammad Zubair Akram et al.

Table-7. Simple correlation (Pearson) of grain yield with other traits

\begin{tabular}{|c|c|c|c|c|c|c|c|c|c|c|}
\hline Parameters & $\begin{array}{c}\text { Grain } \\
\text { Yield }\end{array}$ & $\begin{array}{c}\text { Harvesting } \\
\text { Index }\end{array}$ & $\begin{array}{l}\text { Plant } \\
\text { Height }\end{array}$ & $\begin{array}{c}\text { Plant } \\
\text { biomass }\end{array}$ & Carotenoids & $\begin{array}{l}\text { Root } \\
\text { length }\end{array}$ & $\begin{array}{l}\text { Days to } \\
\text { flowering }\end{array}$ & $\begin{array}{c}\text { Days to } \\
\text { maturity }\end{array}$ & $\begin{array}{c}\text { Total } \\
\text { chlorophyll } \\
\text { contents }\end{array}$ & $\begin{array}{l}\text { Panicle } \\
\text { length }\end{array}$ \\
\hline Harvesting index & $0.7669 * *$ & & & & & & & & & \\
\hline Plant height & $0.4087 * *$ & -0.0658 & & & & & & & & \\
\hline Plant biomass & $0.6324 * *$ & 0.0237 & 0.7919 & & & & & & & \\
\hline Carotenoids & -0.2418 n.s. & 0.0436 & -0.425 & -0.4168 & & & & & & \\
\hline Root length & -0.1916 n.s. & -0.0226 & -0.3139 & -0.2765 & 0.5012 & & & & & \\
\hline Days to flowering & -0.3034 n.s. & 0.0101 & -0.3792 & -0.5028 & -0.042 & 0.000 & & & & \\
\hline Days to maturity & -0.313 n.s. & -0.3839 & 0.2896 & 0.0096 & -0.145 & -0.4038 & -0.0039 & & & \\
\hline Total chlorophyll contents & -0.2661 n.s. & -0.1129 & -0.1108 & -0.2494 & 0.621 & 0.5275 & -0.2121 & 0.0258 & & \\
\hline Panicle length & -0.1423 n.s. & -0.247 & -0.2008 & -0.06 & 0.043 & 0.0591 & -0.2156 & 0.3022 & 0.2253 & \\
\hline 1000-grain weight & -0.268 n.s. & 0.0664 & -0.1796 & -0.4797 & -0.0733 & 0.0226 & 0.5429 & -0.037 & 0.123 & -0.1553 \\
\hline
\end{tabular}

As a result, the selection on the bases of these traits for the improvement of grain yield are not useful. The rate of production of lateral branches directly interlinked with genotype and environmental conditions (SosaZuniga, 2017). Quinoa is preferred for cultivation worldwide due to its high nutritional profile even under varying environment (Sun et al., 2014).

\section{Conclusion}

Ten lines (Q4, Q6, Q9, Q11, Q15, Q22, Q24, Q30, Q45 and Q126) seem to be high yielder than standard check variety UAFQ-7 that are originated from New Mexico and Peru. Finding new germplasm having higher yield potential than the check variety will strengthen the popularity of new crops under the agroclimatic conditions of Faisalabad-Pakistan. This study shows that quinoa has higher adaptive potential for any agro-ecological conditions.

\section{Disclaimer: None. \\ Conflict of Interest: None. \\ Source of Funding: None.}

\section{References}

Basra SMA, Iqbal S and Afzal I, 2014. Evaluating the response of nitrogen application on growth, development and yield of quinoa genotypes. Int. J. Agric. Biol. 16: 886-892.

Bazile D, Pulvento C, Verniau A, Al-Nusairi MS, Ba D, Breidy J, Hassan L, Mohammed MI, Mambetov O and Otambekova M, 2016. Worldwide Evaluations of Quinoa: Preliminary Results from Post International Year of Quinoa FAO Projects in Nine
Countries. Front. Plant Sci. 7: 850.

Bertero HD, Vega AJ, Correa G, Jacobsen SE and Mujica A, 2004. Genotype and genotype-by-environment interaction effects for grain yield and grain size of quinoa (Chenopodium quinoa Willd.) as revealed by pattern analysis of international multi-environment trials. Field Crops Res. 89: 299-318.

Bhargava A, Shukla S and Ohri D, 2006. Chenopodium quinoa: an andean perspective. Indust. Crops and Prod. 23: 73-87.

Bhargava A, Shukla S and Ohri D, 2008. Implications of direct and indirect selection parameters for improvement of grain yield and quality components in Chenopodium quinoa Willd. Int. J. Plant Protec. 2: 184-191.

Brend Y, Galili L, Badani H, Hovav R and Galili S, 2012. Total phenolic content and antioxidant activity of red and yellow quinoa (Chenopodium quinoa Willd.) seeds as affected by baking and cooking conditions. Food Sci. Nutr. 3: 1150-1155.

Bruinsma J, 1963. Chem. Weekbl. 59: 599.

Curti RN, Dela-Vega AJ, Andrade AJ, Bramadi SJ and Bertero HD, 2016. Adaptive responses of quinoa to diverse agro ecological environments along an altitudinal gradient in North West Argentina. Field Crops Res. 186: 10-18.

Fghire R, Ali OI, Anaya F, Benlhabib O, Jacobsen SE and Wahbi S, 2013. Protective antioxidant enzyme activities are affected by drought in quinoa (Chenopodium quinoa Willd). J. Biol. Agric. Health. 3: 4-11.

Gregory PJ, 2006. Plant Roots: Growth, Activity and Interaction with Soils, Blackwell Publishing Ltd, Oxford, UK, pp. 216.

Hellin J and Higman S, 2005. Crop Diversity and Livelihood Security in the Andes. Devel. Practice. 15: 165-174. 
Iqbal S, Basra SMA, Afzal I and Wahid A, 2017. Exploring potential of well adapted quinoa lines for salt tolerance. Int. J. Agric. Biol. 19: 933-940.

Iqbal S, Basra SMA, Afzal I, Wahid A, Saddiq MS, Hafeez MB and Jacobson SE, 2019. Yield potential and salt tolerance of quinoa on salt-degraded soils of Pakistan. J. Agric. Crop Sci. 205(1): 13-21.

Jacobsen SE, Mujica A and Ortiz R, 2003. The Global Potential for Quinoa and Other Andean Crops. Food Rev. Int. 19: 139-148.

Khosravinejad F, Heydari R and Farboodnia T, 2008. Effects of salinity on photosynthetic pigments, respiration, and water content in two barley varieties. Pak. J. Biol. Sci. 11: 2438-2442.

Lees DH and Francis FJ, 1971. Quantitative methods for anthocyanins. VI. Flavonols and anthocyanins in fresh cranberries. J. Food Sci. 36: 1056.

Li H, Rasheed A, Hickey LT and He Z, 2018. Fastforwarding genetic gain. Trends in Plant Sci. 23: 184186.

Meloni DA, Gulotta MR and Martínez CA, 2008. Salinity tolerance in Schinopsis quebracho colorado: seed germination, growth, ion relations and metabolic responses. J. Arid Environ. 72: 1785-1792.

Parida AK and Das AB, 2005. Salt tolerance and salinity effects on plants: a review. Ecotoxicol. Environ. Saf. 60: 324-349.

Pulvento C, Riccardi R, Lavini R, d'Andria R, Iafelice G and Marconi E, 2010. Field trial evaluation of two Chenopodium quinoa genotypes grown under rainfed conditions in a typical Mediterranean environment in South Italy. J. Agro. Crop Sci. 196: 407-411.

Radhouane PL, 2018. Why don't adapt Tunisian agriculture to climate change? Mitigation et adaptation. Int. J. Environ. Sci. 7: 1615-1630.

Rashid N, Basra SMA, Shahbaz M, Iqbal S and Hafeez MB, 2018. Foliar Applied Moringa Leaf Extract Induces Terminal Heat Tolerance in Quinoa. Int. J. Agric. Biol. 20: 157-164.

Razaq M, Zhang P and Shen HL, 2017. Influence of nitrogen and phosphorous on the growth and root morphology of Acer mono. PloS One. 12: e0171321.

Repo-Carrasco R, Espinoza C and Jacobsen SE, 2003. Nutritional value and use of the Andean crops quinoa (Chenopodium quinoa) and kaniwa (Chenopodium pallidicaule). Food Rev. Int. 19: 179-189.

Ruiz KB, Biondi S, Oses R, Acuña-Rodríguez IS, Antognoni F, Martinez-Mosqueira EA, Coulibaly A, Canahua-Murillo A, Pinto M and Zurita-Silva A, 2014. Quinoa Biodiversity and Sustainability for Food Security under Climate Change, A Review.
Agro. Sust. Devel. 34: 349-359.

Sajjad A, Munir H, Ehsanullah AAS, Tanveer M and Rehman A, 2014. Growth and development of Chenopodium quinoa genotypes and different sowing dates. J. Agric. Res. 52: 535-546.

Shabala L, Mackay A, Tian Y, Jacobsen SE, Zhou D and Shabala S, 2012. Oxidative stress protection and stomatal patterning as components of salinity tolerance mechanism in quinoa (Chenopodium quinoa). Physio. Planta. 146: 26-38.

Sosa-Zuniga V, Brito V, Fuentes F and Steinfort U, 2017. Phenological growth stages of quinoa (Chenopodium quinoa) based on the BBCH scale. Ann. App. Biol. 171: 117-124.

Steel RGD, Torrie JH and Dicky DA, 1997. "Principles and procedures of statistics: A biological approach." $3^{\text {rd }}$ Eds. Mcgraw Hill Inc. Book Co. N.Y. USA. pp. 352-358.

Sun Y, Liu F, Bendevis M, Shabala S and Jacobsen SE, 2014. Sensitivity of two quinoa (Chenopodium quinoa Willd.) varieties to progressive drought stress. J. Agron. Crop Sci. 200: 12-23.

Vega-Galvez A, Miranda M, Vergara J, Uribe E, Puente L and Martínez EA, 2010. Nutrition facts and functional potential of quinoa (Chenopodium quinoa Willd.), an ancient Andean grain: A review. J. Sci. Food Agric. 90: 2541-2547.

Waisel Y, Eshel A, Kafkafi U, Waisel Y and Eshel A, 2002. Functional diversity of various constituents of a single root system. In "Plant Roots: The Hidden Half," edited by Waisel, Y., A. Eshel, U. Kafkafi, Ed 3. Marcel Dekker, New York, pp 157-175.

Zurita-Silva A, Fuentes F, Zamora P, Jacobsen SE and Schwember AR, 2014. Breeding Quinoa (Chenopodium Quinoa Willd.): Potential and Perspectives. Mol. Breed. 34: 13-30.

\section{Contribution of Authors}

Akram MZ: Planned \& conducted experiment, data analysis \& prepared first draft of article Basra SMA: Planned the experiment and gave final approval of manuscript

Hafeez MB \& Khan S: Data analysis and manuscript editing

Nazeer S \& Zahra N: Data collection \& analysis Iqbal S \& Saddiq MS: Literature review and manuscript editing 